\title{
Management of subglottic stenosis: experience from two centres
}

\author{
R E QUINEY, M G SPENCER, C M BAILEY, J N G EVANS, AND J M GRAHAM \\ Royal Ear Hospital, University College Hospital, and Hospital for Sick Children, London
}

SUMMARY The incidence of subglottic stenosis in children has risen rapidly in the last 20 years as more advanced techniques enable younger preterm neonates to survive. There has been a similar rise in the number of different surgical methods devised to alleviate the stenosis; success has been claimed for each technique. The importance of normal laryngeal growth throughout an often protracted period of surgical intervention may, however, have been underestimated. This study analyses the data from two units in London over the last five years and assesses retrospectively the benefit of different surgical approaches.

Improved ventilation methods for neonates in intensive care units, while saving the lives of children with bronchopulmonary dysplasia and hyaline membrane disease, have resulted in prolonged periods of intubation. Acquired subglottic stenosis at the level of the cricoid cartilage ring, which is the narrowest and most unyielding part of the airway, has been reported, with incidence ranging from $1-8 \% .^{1-3}$

Subglottic stenosis of congenital origin is less common by comparison and can only be diagnosed with certainty in a child before its first intubation.

Children who develop subglottic stenosis are subject to prolonged periods in hospital and are often referred to specialist units. Tracheostomy is often required and management is not without complication, including death from lumen obstruction or misplacement of the tube. (Fearon and Cotton in 1974 reported a $24 \%$ mortality in their patients with subglottic stenosis as a direct result of having a tracheostomy. ${ }^{4}$ )

Children with subglottic stenosis may have few other problems after leaving the neonatal intensive care unit, yet subsequently many different groups of specialists (ear, nose, and throat surgeons, speech therapists, paediatricians, physiotherapists, and social workers) may be involved in the management of this single complication for the next five years.

The factors that may cause subglottic stenosis (prolonged intubation, method of intubation, type of endotracheal tube used, and associated infection) have been reviewed previously. ${ }^{56}$

In this paper we have reviewed all the cases of subglottic stenosis seen at The Hospital for Sick Children, Great Ormond Street, and University
College Hospital, London, over the last five years. Most of these patients have been referred from other units in the United Kingdom, predominantly the south east.

We have looked at the management of these children in the two different units and assessed the success of each method of treatment.

\section{Methods and materials}

All cases of subglottic stenosis referred to the ear, nose, and throat departments of the two hospitals from 1980 onwards have been reviewed. Clinical details, method and length of intubation, and need for tracheostomy were noted for each patient. Information from referring hospitals was not always complete. The severity of stenosis at diagnosis by direct laryngoscopy and at subsequent observation was noted. Details of timing and method of surgical repair together with the outcome were collected. Some specific problems in the management of these patients are mentioned.

\section{Clinical observations}

A total of 110 patients with subglottic stenosis were seen in the two units from 1980 onwards. Eighty eight were from the Hospital for Sick Children and 22 from University College Hospital. Hospital records were available for only $80(73 \%)$ of these, and this forms the basis of the study. Of the 80 patients, 73 were referred from other hospitals. All patients at the Hospital for Sick Children were referred from elsewhere, while patients at University College 
Hospital were referred from both that hospital's neonatal unit and other centres.

Average gestational age of the children was 29.7 weeks, with a range of $24.4-41$ weeks and a median of $28 \cdot 1$ weeks.

Average birth weight was $1290 \mathrm{~g}$, with a range of $650-3400 \mathrm{~g}$ and a median of $1160 \mathrm{~g}$.

All but five of the patients were intubated at or soon after birth. Fifty patients were intubated with an oral tube and 19 with a nasotracheal tube. Portex oral shouldered (Coles) tubes were used in 34 patients, parallel sided oral tubes in 16 , and parallel sided nasal tubes in 19. The type of tube used in six patients was unknown. The duration from birth to diagnosis of subglottic stenosis at direct laryngoscopy varied from three days to 18 months, with a median time of 10 weeks. This wide range can be divided into three separate populations.

(1) Fifty five children presented as failures of attempted extubation within three months of birth while still in the neonatal unit.

(2) A second group comprised 18 children who presented between three months and one year. Having been successfully extubated and discharged from the neonatal unit, they presented later with stridor precipitated usually by an upper respiratory tract infection.

(3) In a third group, comprising seven children, diagnosis was made at over 1 year of age. The cause of stridor in these seven patients is shown in Table 1.

Of the 80 cases, four were considered to be true cases of congenital subglottic stenosis, the rest (76) being acquired after intubation.

\section{Management without a tracheostomy}

Thirteen patients did not require a tracheostomy.
These patients had a greater than average birth weight (1830 g) and an older than average gestational age (30.4 weeks) than the others. Their average age at presentation was 25 weeks. Of these, eight were treated with a cricoid split operation as described by Cotton $e t \mathrm{al}^{7}$ and were then successfully extubated one to four weeks later. The remaining five had no surgical intervention but were observed by periodic direct laryngoscopy and were extubated two to four weeks after their initial diagnosis.

None have had a recurrence of their stenosis. All have shown normal speech development. No complications of surgical intervention have been found.

\section{Management with tracheostomy}

The remaining 67 patients required a tracheostomy. Excluding the seven late presenters (Table 1) who form a rather specialised group, the median age at tracheostomy was 11 weeks, with a range of 1 to 78 weeks. Tracheostomy involved a skin crease incision, upward retraction or division of the thyroid isthmus, and vertical incision of the anterior tracheal wall below the first tracheal ring. Nylon stay sutures were used in each edge of the cut trachea and brought out of the wound and taped to the child's chest, thereby enabling the trachea to be opened by traction on these sutures either at the first tracheostomy tube change (normally at 7 days) or in the event of a displaced tracheostomy tube. Tracheostomy tubes used varied between the units but included Great Ormond Street pattern, Portex, and Shiley tubes. The advantage of the Shiley tube is its large plastic flange around the entry hole that prevents the child's chin drooping down and obstructing the lumen. Great Ormond Street tubes are now available with this modification. Silver Alder

Table 1 Diagnosis of subglottic stenosis in seven patients, presentation at greater than 1 year of age

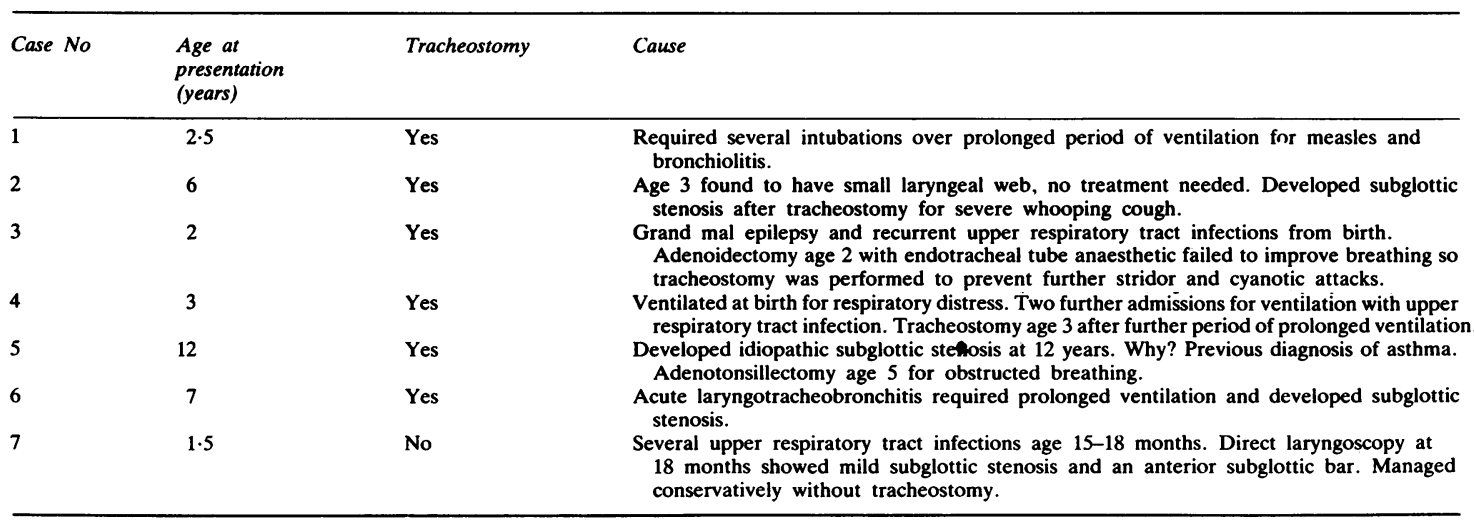


Hey tubes were rarely used because they tended to produce greater stomal irritation, they were less comfortable, and correct positioning of the tube fenestra was difficult in small infants.

The size of the subglottic lumen was measured accurately at direct laryngoscopy at the time of tracheostomy by sizing the lumen with known diameter endotracheal tubes, though this was performed in only 30 patients. The lumen varied from a maximum diameter of $2.3 \mathrm{~mm}$ to a stenosis so tight that air was unable to escape up from the trachea. The average lumen diameter was $1.6 \mathrm{~mm}$.

After tracheostomy the children were generally allowed home with adequate home support. While on the ward mothers were taught to change tracheostomy tubes, use suction equipment, and provide adequate humidification. This training period could last several weeks. Home support involved provision of portable suction equipment, spare tracheostomy tubes, a home help if required, a tracheostomy care sister based between the community and the ward, and contact with the charity organisation Aid for Children with Tracheostomies.

Patients were assessed by direct laryngoscopy about every three months, lumen size being measured and compared with previous records. In this way some record of laryngeal growth, if any, was made.

In this group of 67 patients who needed a tracheostomy 20 had a cricoid split operation before tracheostomy while still intubated. This procedure was considered unsuccessful at further direct laryngoscopy and the patients therefore required a tracheostomy. This group of 20 had a smaller average birth weight and gestational age than those patients described above who were successfully treated with a cricoid split, thereby avoiding a tracheostomy.

Of the 67 patients with a tracheostomy, 32 have had repair of the subglottic region attempted, eight are awaiting a repair at a suitable age, 27 have been managed conservatively, and five patients have died during treatment.

In the 32 cases that have had laryngeal reconstruction the following occurred.

(1) Attempt at surgical repair when the child with tracheostomy was 18-24 months old. This timing allows a suitable period for normal laryngeal growth to occur, which can be monitored at serial laryngoscopy. It also makes the planned surgery technically easier than if operating at a much younger age. Of the 32 patients, 17 had a castellated laryngotracheoplasty as described by Evans ${ }^{8}$ and 15 had an anterior rib graft to a vertically split larynx and trachea as described by Cotton. ${ }^{9}$ Of the 32 patients, only 20 were successfully decannulated
Table 2 Summary of surgical procedures performed in 12 patients requiring more than one major attempt at subglottic reconstruction

\begin{tabular}{lll}
\hline $\begin{array}{l}\text { Operative sequence } \\
\text { patients }\end{array}$ & $\begin{array}{l}\text { Outcome } \\
\text { to date } \\
\text { (No decan- } \\
\text { nulated) }\end{array}$ \\
\hline $\begin{array}{l}\text { Laryngotracheoplasty, rib graft } \\
\text { Laryngotracheoplasty, two } \\
\text { rib grafts }\end{array}$ & 4 & 2 \\
$\begin{array}{l}\text { Two rib grafts } \\
\text { Laryngotracheoplasty and } \\
\text { multiple lasering } \\
\text { to remaining stenosis }\end{array}$ & 2 & 1 \\
$\begin{array}{l}\text { Laryngofissure and excision } \\
\text { of scar tissue and stenting }\end{array}$ & 2 & 1 \\
$\begin{array}{l}\text { Repair of tracheo-oesophageal } \\
\text { fistula and subglottic stenosis }\end{array}$ & 2 & 2 \\
\hline
\end{tabular}

In some of the revision rib grafts both an anterior and posterior rib graft were inserted.

(Most of these patients have also had numerous endoscopic procedures. sometimes with removal of granulations or scar tissue with cupped forceps, diathermy, dilation, or laser vaporisation. The Table only indicates the main operations performed.)

without further surgical intervention. This occurred six to 51 weeks after operation.

(2) Repeat attempts at laryngeal reconstruction generally three or more months after the first repair for a continuing inadequate airway as assessed by direct laryngoscopy. The summary of these operations and their subsequent outcome are shown in Table 2 .

In 27 patients no surgical intervention after tracheostomy was needed. Good laryngeal growth enabled decannulation to occur four to 26 months after tracheostomy.

These figures may suggest poor success for all surgical procedures attempted. It is important to point out that of the 20 patients still not decannulated, eight have not reached the age for surgical intervention and four have only recently had an operation and are planned for decannulation in the near future.

Five of our children with subglottic stenosis died. All had had tracheostomies. Four died at home, the causes of death being a displaced tracheostomy tube, a tracheostomy tube blocked with viscid mucus and a tracheal granuloma, and two unexplained cot deaths with unremarkable postmortem findings. The fifth death occurred in a 6 month old child who developed an unresponsive bilateral lung consolidation caused by a mixed infection of klebsiella and pseudomonas species despite intensive care, including ventilation.

\section{Discussion}

Only University College Hospital has a set of 
patients indigenous to its own hospital. The reported incidence of subglottic stenosis varies from $1-8 \% .{ }^{1-3}$ Over the five years of this study the incidence of subglottic stenosis in children born at the hospital was $1.8 \%$ of all intubated neonates, but of surviving infants the incidence was $2.6 \%$. $^{5}$

The problem of management of these patients has been noted by a number of authors, ${ }^{10} 11$ and we found similar difficulties. Our overall mortality was $5 \%$, which confirms the serious nature of this condition and the need for prevention if possible and correct and meticulous management.

The results should not be interpreted as suggesting there is little statistical difference in success with decannulation between those patients who have an operation and those managed conservatively. These two groups are not comparable; those who are operated on aged 18 months to 2 years are failures of conservative treatment and therefore make a bad risk group in themselves. Although there seems to be no real difference in lumen size at the time of tracheostomy in these two groups, in one group it becomes apparent within 18 months of tracheostomy that laryngeal growth is inadequate to permit decannulation. The reason for this difference is not clear.

Although by the age of 18 months there is mounting pressure from parents to operate, the natural history of these children is not really known. Without a control group, which would be ethically and emotionally unacceptable, an operation's success cannot be clear. The assumption that because the larynx has failed to grow in the last 18 months it is so stenosed that no further adequate growth will occur should at least be questioned.

There seems to be no statistical difference in success between the two main types of operation under review, a castellated laryngotracheoplasty or an interposed rib graft. Trends and ideas often change; five years ago castellated laryngotracheoplasty $^{8}$ was the method of choice, but more recently rib graft repairs have become more common. This study cannot resolve which is the better technique. Both have similar success and complication rates. The rib graft might be simpler to perform but it involves a separate incision to harvest a rib cartilage. Some patients received both operations (Table 2) and yet remain with a tracheostomy. Current thoughts are that the laryngotracheoplasty should be reserved for those cases of congenital subglottic stenosis (or occasionally very mild acquired stenosis) where there has been no accumulation of dense scar tissue secondary to intubation and where there is therefore a good enough cartilage ring structure to permit the castellated incision to be performed.

In most cases of acquired subglottic stenosis the lumen needs to be held open with a strut of rib cartilage to prevent cicatrical stenosis reforming, as it does if castellation is attempted in scar tissue. In revision operations where an anterior rib graft has failed to provide a satisfactory airway combined anterior and posterior rib grafts may be more successful in widening the subglottic lumen, but possibly at the expense of voice quality; the edges of the arytenoids are moved apart by the rib graft inserted between the edges of the posteriorly split cricoid cartilage and as a result adduction of the vocal cords is impeded.

Our study consists of three age groups at presentation. There does seem to be a correlation between severity of stenosis and age at presentation, the older age groups having a less severe stenosis as might be predicted. Certainly, the most difficult to treat are those of low birth weight and low gestational age who are intubated for long periods and never decannulated. The failure of operative intervention in these patients may reflect poor laryngeal growth in a very scarred larynx.

\section{What treatment policies in future?}

\section{Prevention}

Prevention may not always be possible but there is evidence that frequent and prolonged intubations only make the problem worse. ${ }^{12}{ }^{13}$ The use of oral Portex Coles pattern shouldered tubes always runs the risk of the shoulder part of the tube being impacted into the cricoid ring, giving rapid pressure necrosis to this region despite the widespread use of a post-intubation chest or lateral neck $x$ ray. Impaction should be avoidable if the tip of the tube lies at or above the level of the clavicles on chest $x$ ray. Gould has shown that this can occur within a few hours to give irreversible stenosis. ${ }^{14}$ Other possible preventable causes have been discussed previously. ${ }^{5}$

\section{Tracheostomy-indications and timing}

Once subglottic stenosis has been diagnosed which treatment should be adopted? Having an endotracheal tube in place may cause further irritation and make the stenosis worse. Alternatively, it might allow a period of re-epithelialisation of the necrosed mucosa around a suitable sized stent. The former argues for an early tracheostomy, the latter for prolonged undisturbed intubation. The possibility that perhaps a tracheostomy itself may make a stenosis worse by introducing infection locally has not been confirmed in humans, although the work by Saski et al on dogs concluded that infective chondritis after tracheostomy makes stenosis worse. ${ }^{15}$

In the early presenting group of patients with a primary failure of extubation on the neonatal unit 
tracheostomy is invariably required if a trial of extubation fails after two to three weeks of conservative management when the endotracheal tube is correctly sited and ideally undisturbed. Other problems such as chest infections should be resolved before this undisturbed period, but in practice the chest problems may fail to improve without a tracheostomy. A cricoid split does not usually help these patients.

Certainly, this study suggests that in those patients who present late (groups 2 and 3 above) with a mild stenosis further intubation for a number of weeks may allow decannulation. The cricoid split operation has had some excellent results in such patients and as it is a fairly small procedure it is to be recommended where conservative treatment is failing. An anterior cricoid split alone followed by intubation for a further 10 days gives time for stabilisation and epithelialisation and should be performed before any decision to proceed to tracheostomy.

\section{Management after tracheostomy}

In those patients who require tracheostomy every effort must be made to enable the patient to go home and lead as near normal a life as possible. Support by trained staff within the community has been essential to this. Direct laryngoscopy should be performed every three or four months in those with a mild stenosis and in whom good laryngeal growth is seen, but this seems unnecessarily frequent in those with a tight stenosis. The lumen size should always be recorded by measuring with known size endotracheal tubes, nasal catheters, or bronchoscopes. Our results suggest that in the 32 patients who had a reconstructive operation the best chance of decannulation came with the first attempt at laryngeal repair at 18 to 24 months. We would recommend the use of per- and postoperative antibiotics to prevent infective chondritis and further scar tissue developing. The use of different stents has not been assessed; a tight fitting stent may cause pressure necrosis by itself so that when the stent is removed the lumen collapses inwards and then further scar tissue accumulates. In the Hospital for Sick Children a coiled piece of silastic sheeting was used to act as a self adjusting stent, expanding and contracting to fit the lumen without causing pressure necrosis.

\section{References}

1 Strong RM, Passy V. Endotracheal intubation: complications in neonates. Arch Otolaryngol 1977:103:329-35.

2 Papisidero MJ, Pashley NRT. Acquired stenosis of the upper airway in neonates. Ann Otol Rhinol Laryngol 1980;89:512.

3 Ratner I, Whitfield J. Acquired subglottic stenosis in the very low birthweight infant. Am J Dis Child 1983;137:40-3.

${ }^{4}$ Fearon B, Cotton R. Surgical correction of subglottic stenosis of the larynx in infants and children. Progress report. Ann Otol Rhinol Laryngol 1974;83:428-31.

${ }^{5}$ Quiney R, Gould S. Subglottic stenosis: a clinicopathological study. Clin Otol 1985;10:315-27.

6 Gould SJ, Graham JM. Acquired subglottic stenosis in neonates. Clin Otol 1985;10:299-302.

7 Cotton RT, Seid AS. Management of the extubation problem in the premature child. Anterior cricoid split as an alternative to tracheostomy. Ann Otol Rhinol Laryngol 1980;89:508-11.

${ }^{8}$ Evans JNG, Todd GB. Laryngotracheoplasty. J Laryngol Otol 1974;88:589-97.

${ }^{9}$ Cotton RT. Management of subglottic stenosis in infancy and childhood. Ann Otol Rhinol Laryngol 1978;87:649-57.

10 Fearon B, Cotton RT. Subglottic stenosis in infants and children. The clinical problem and experimental surgical correction. Ann Otol Rhinol Laryngol 1971;80:669-77.

"Hollinger PH, Kutnick SL, Schild JA, et al. Subglottic stenosis in infants and children. Ann Otol Rhinol Laryngol 1976;85: 591-9.

12 Hawkins DB. Hyaline membrane disease of the neonate prolonged intubation in management: effect on the larynx. Laryngoscope 1970;88:201-24.

13 Rasche $\overline{R F H}$, Kuhns LR. Histopathological changes in airway mucosa of infants after endotracheal intubation. Pediatrics 1972;50:632-7.

14 Gould SJ, Howard S. The histopathology of the larynx in the neonate following endotracheal intubation. J Pathol 1985; 146:301-11.

Is Saski CC, Horiuchi M, Koss N. Tracheostomy related subglottic stenosis. Bacteriological pathogenesis. Laryngoscope 1979;89: 857-65.

Correspondence to Mr R E Quiney, Royal Ear Hospital, Huntley Street, London WC1.

Received 8 April 1986 